\title{
Estilo de liderazgo del director y clima escolar en un establecimiento educacional al implementar un programa externo de intervención: Validación de instrumento
}

\author{
Director's leadership style and school climate in an educational establishment by \\ implementing an external intervention program: Instrument validation
}

\author{
Rafael Arancibia Rojas ${ }^{a}$, Eugenio Chandía Muñoz ${ }^{b}$ \\ ${ }^{a}$ Universidad Católica Silva Henríquez. \\ rarancibia@ucsh.cl \\ ${ }^{b}$ Centro de Investigación Avanzada en Educación, Universidad de Chile. \\ echandia@dim.uchile.cl
}

\begin{abstract}
RESUMEN
El presente artículo presenta un estudio realizado en dos etapas. La primera etapa corresponde a una revisión bibliográfica para conocer las condiciones internas de los establecimientos educacionales que afectan la implementación y sostenibilidad de los procesos de mejoramiento propuestos por un programa de asesoría técnica educativa. La segunda etapa considera la construcción y validación de un instrumento que permite levantar esta información. Se trata de un estudio cuantitativo que incluye una técnica estadística de reducción de datos que permite reducir el número de variables que describen un concepto. Los resultados proporcionan variables capaces de describir el estilo de liderazgo del director y el clima escolar en un establecimiento educacional al implementar un programa externo de intervención.
\end{abstract}

Palabras claves: mejora educativa, desarrollo profesional docente, asesoría externa, condiciones institucionales, confianza.

\section{ABSTRACT}

The present article describes a study carried out in two stages. The first corresponds to a bibliographic review to identify the internal conditions of the educational establishments that affect the implementation and sustainability of the improvement processes proposed by a specialized advisory program for education. The second stage considers the construction and validation of an instrument that allows this information to be collected. It is a quantitative study that includes a modification of the data capacity that allows reducing the number of variables that describe a concept. The results provide variables that allow describing the director's leadership style and school climate in an educational establishment by implementing an external intervention program.

Key words: educational improvement, professional development, external advisory, institutional conditions, trust. 


\section{INTRODUCCIÓN}

Desde principios de los años 90 que las políticas educativas en Chile buscan establecer procesos de mejora en las escuelas a través de programas externos de asesoramiento. En este contexto aparecen algunas iniciativas como el programa P900, la estrategia LEM y el plan de asistencia técnica a escuelas críticas; desarrolladas principalmente por universidades chilenas. A partir del año 2008, con la aprobación de la ley de Subvención Escolar Preferencial, los programas de asistencia técnica educativa (ATE) pasan a ser la herramienta central de las políticas educativas de mejoramiento.

Los estudios desarrollados a la fecha se han enfocado en conocer características y efectos de los programas de asistencia técnica en instituciones escolares y en su contribución con procesos de mejora sostenibles en el tiempo (Bellei, Osses, \& Valenzuela, 2010; Marchant \& Salin, 2009; Ríos \& Villalobos, 2016). Estas investigaciones coinciden en la importancia de las condiciones internas de los establecimientos educativos en la implementación y sostenibilidad de los procesos de mejora que proponen los programas de asesoramiento. En este sentido, algunos de los elementos fundamentales son el estilo de liderazgo del director y el clima escolar (López Yáñez, 2010).

Utilizando un instrumento cuantitativo, Tshcannen-Moran (2009) recoge información acerca de las condiciones internas de los establecimientos educativos, en particular del estilo de liderazgo del director y la confianza en las relaciones interpersonales, este último relacionado con el clima escolar; que de acuerdo con la literatura (Hernández-Castilla et al., 2013; Hoy \& Sweetland, 2001; Sweetland \& Hoy, 2000; Ríos \& Villalobos, 2016; Tschannen-Moran, 2004, 2009, 2013), impactan en el logro de objetivos compartidos y la implementación efectiva de programas externos de intervención.

Dado que en la literatura nacional no se encuentran instrumentos cuantitativos validados para levantar esta información, el objetivo del presente trabajo es diseñar un instrumento para conocer el estilo de liderazgo del director y el clima escolar en la organización educativa. Este procedimiento incluye la construcción y validación de un instrumento ajustado de acuerdo con los trabajos de Tshcannen-Moran (2004, 2009, 2013) para recoger información en las siguientes dimensiones:

1. Estilo de liderazgo del director,

2. Confianza en las relaciones interpersonales,

3. Estructura organizacional de la escuela $y$,

4. Grado de satisfacción y motivación laboral de los docentes.

Para los programas de asistencia técnica educativa (ATE) resulta relevante conocer las condiciones internas de los establecimientos escolares que apoyarán, de forma tal que puedan anteponerse a escenarios que atenten contra una efectiva implementación y sostenibilidad de los procesos de mejora propuestos.

\section{MARCO REFERENCIAL Y CONCEPTUAL}

Tradicionalmente la escuela ha sido una institución cerrada, inserta en una estructura burocrática de organización. Su principal conexión con el exterior la constituyen los sistemas públicos de inspección, supervisión y control, y en general, pocos países han 
tomado la decisión de intervenirlas. La forma de intervención varía, por ejemplo, en Estados Unidos e Inglaterra las entidades de intervención son externas al Ministerio de Educación. En Nueva Zelanda y Suecia, en cambio, los servicios de apoyo son entregados por las entidades públicas. Respecto al sentido y objetivo de los programas externos de intervención, en general son entendidos como recursos complementarios y funcionales a las políticas nacionales de fortalecimiento, donde la decisión de apoyar a determinadas escuelas vulnerables corresponde principalmente a las autoridades educacionales (Bellei, Osses \& Valenzuela, 2010).

En Chile, hace tres décadas existe la intención de vincular e intervenir los establecimientos escolares con programas externos que entreguen asistencia técnica educativa. Algunos de los programas más relevantes en el país son el P900 entre 1990 y 2000 , enfocado en dar un apoyo técnico y material al 10\% de escuelas pobres con bajos resultados en el SIMCE de matemática y lenguaje; el plan de asistencia técnica educativa a escuelas críticas entre 2002 y 2005 , enfocado en intervenir 66 escuelas con bajos resultados de aprendizaje, altos índices de repitencia y deserción y, la estrategia LEM entre el 2004 y el 2005, que buscaba impactar a un mayor número de escuelas básicas subvencionadas por medio de una propuesta de implementación curricular en matemática y lenguaje (Sotomayor, 2006).

El año 2008 se aprueba la Ley de Subvención Escolar Preferencial (SEP) con el fin de asegurar un servicio educativo de calidad para los alumnos de los establecimientos educativos que reciben subvención estatal. Esta ley, además de entregar recursos a los establecimientos para cumplir con las estrategias de mejoramiento definidas autónomamente, permite la contratación de servicios de asistencia educativa bajo la figura de apoyos externos que permiten a las escuelas alcanzar las metas de mejoramiento asumidas ante la autoridad. De esta forma, los servicios de asistencia técnica educativa (ATE), se constituyen como parte de una estrategia de la política educativa de la época, que tiene por función prestar una asesoría técnico pedagógica externa y directa a las escuelas, a través de la instalación de procesos de mejoramiento, que se espera sean sostenibles en el tiempo. En este contexto, los programas externos de asesoramiento educativo se convierten en la herramienta central de las políticas de mejoramiento escolar en Chile (Bellei et al., 2010).

Respecto de los servicios de asesoría técnica y los procesos de mejoramiento escolar, la literatura nacional e internacional sugiere que el resultado de una ATE depende tanto de las características del programa como de la realidad específica de cada escuela. El trabajo de López, Ahumada, Olivares \& González (2012) estudia las condiciones internas de las escuelas que hacen posible gestionar un cambio hacia la mejora. Sin embargo, a nivel latinoamericano casi no existen estudios específicos respecto de la sustentabilidad de estos procesos y su relación con las condiciones que ofrecen los establecimientos (Valenzuela \& Allende, 2014).

En general, sobre los entornos que contribuyen a la implementación y sostenibilidad de los procesos de mejora propuestos por una ATE, López (2010) enfatiza que son las condiciones internas del establecimiento educativo la base fundamental sobre la cual los cambios son construidos de manera estable y efectiva. Por lo tanto, lograr que los procesos de mejora se establezcan y mantengan en el tiempo, depende en gran parte de las características propias de la organización escolar (Bellei et al., 2010; Espíndola \& Silva, 2009; Marchant \& Salin, 2009). Donde sobresalen el estilo de liderazgo del director y el clima escolar (Ríos \& Villalobos, 2016). 
En este sentido, el estilo de liderazgo del director puede contribuir positivamente cuando tiene características de liderazgo distribuido, promoviendo el diálogo y las decisiones compartidas; enfatizando una visión de liderazgo alejada de las individualidades y promoviendo en todos los miembros de la comunidad la iniciativa y la innovación; generando un clima de buena convivencia y compromiso con la labor a realizar; apoyando permanentemente a los docentes en las exigencias que el trabajo de asesoría requiere y garantizando los tiempos y espacios de trabajo (López, 2010; Ríos \& Villalobos, 2016).

Además, dentro de las condiciones institucionales que facilitan la implementación de los procesos de mejora, se considera un clima escolar que genere satisfacción en los docentes. De acuerdo con López (2010), en los procesos de mejoramiento sostenibles se logra crear una cultura de aprendizaje y desarrollo profesional continuo a través de la colaboración y el apoyo mutuo, los que a su vez resultan de un clima escolar que promueve el compromiso de todos. Por el contrario, aquellos elementos propios de las escuelas que dificultan la implementación de los procesos de mejora son, entre otros, un clima escolar caracterizado por la desconfianza, la división, la desmotivación y falta de compromiso de los docentes, un equipo directivo poco comprometido y bajas expectativas de logro entre los actores de la comunidad (Hernández-Castilla, Murillo \& Martínez-Garrido, 2013).

Una revisión de la literatura dedicada al estudio de las condiciones internas de los establecimientos escolares en el plano del liderazgo y el clima escolar (Hernández-Castilla et al., 2013; Hoy \& Sweetland, 2001; Sweetland \& Hoy, 2000; Ríos \& Villalobos, 2016; Tschannen-Moran, 2004, 2009, 2013) permite sintetizar y describir las dimensiones que más impactan en la efectiva implementación de procesos de mejora que sean sostenibles en el tiempo:

1. Confianza: En las relaciones interpersonales basadas en la confianza es posible encontrar altas expectativas acerca de la competencia profesional del otro, una alta estima personal por el otro, integridad y respeto (Razeto, 2016; Tshcannen-Moran, 2009, 2013). Esta confianza en las relaciones interpersonales genera un clima escolar positivo de trabajo, una alta satisfacción percibida por los docentes y relaciones más horizontales entre los trabajadores y sus líderes (HernándezCastilla et al., 2013; López Yáñez, 2010; Tschannen-Moran, 2009, 2013).

2. Orientación del director (o estilo de liderazgo): En la literatura se reconocen dos orientaciones: burocrática y profesional. La orientación burocrática se caracteriza principalmente porque los líderes concentran el poder de decisión, tienen poca consideración por la capacidad profesional de los docentes y los disciplinan mediante el cumplimiento de las reglas de la organización. Estos líderes sobrevaloran las reglas y las políticas de control. Por otro lado, los líderes escolares con una orientación profesional tienen en mayor estima la experiencia profesional de los docentes, creen más en sus capacidades y les dan más autonomía para ejercer el juicio profesional. Y pese a que existen políticas y procedimientos prescritos, se consideran como guías flexibles de la práctica, más que como un conjunto de procesos rígidos que deben seguirse bajo cualquier circunstancia (López et al., 2012; Tschannen-Moran, 2009, 2013).

3. Satisfacción y motivación de los docentes: Como parte de las condiciones necesarias que se presentan dentro de los establecimientos educacionales para alcanzar objetivos compartidos, la satisfacción y motivación laboral de los 
docentes resultan relevantes (López et al., 2012; Tschannen-Moran, 2009, 2013). En particular, cuando el líder posee una orientación burocrática, la satisfacción laboral de los profesores es baja. Por el contrario, cuando el líder tiene una orientación profesional, es probable que los docentes se sientan más satisfechos con el trabajo, más motivados por alcanzar objetivos compartidos y más comprometidos con los objetivos comunes. Para alcanzar objetivos compartidos es necesaria la motivación de quienes participan. En este caso, para implementar efectivamente un programa externo de intervención, es necesaria la motivación de los profesionales que forman parte de la comunidad (Ríos \& Villalobos, 2016).

4. Estructura de la organización: La estructura organizacional que adopta la escuela también juega un rol fundamental en el logro de objetivos compartidos y en la adaptación a los cambios sugeridos por programas externos de intervención (Hoy y Sweetland, 2000, 2001; Ríos \& Villalobos, 2016; López et al., 2012; TschannenMoran, 2004, 2009, 2013). Esta estructura es el resultado de la interacción y disposición de diferentes elementos, entre los que resultan más predominantes: el grado de confianza en las relaciones interpersonales (en particular la confianza que tiene el director en los profesores) y la orientación del director (Tschannen-Moran, 2009). Esto significa que cuando un líder escolar no confía en sus profesores, en cuanto a capacidad profesional e integridad, ejecuta prácticas para supervisar, controlar y normalizar el trabajo de los profesores (Tschannen-Moran, 2009). Reforzando así una desconfianza implícita que se amplía a toda la comunidad, dificultando el trabajo colaborativo y también, el logro de objetivos compartidos (Ríos \& Villalobos, 2016).

\section{MÉTODO}

El diseño de investigación utilizado en este trabajo corresponde a un diseño cuantitativo no experimental. Para esto se constituyó una muestra no representativa de siete establecimientos escolares, pertenecientes a la Red de Colegios de la Sociedad de Instrucción Primaria de la Región Metropolitana, en los que se aplica dos cuestionarios: uno dirigido a profesores y otro al equipo directivo, ambos a mitad de año. En cuatro de estos siete colegios hace más de un año se implementa un programa de desarrollo profesional docente de la Universidad de Chile, cuyo propósito es impactar en las prácticas docentes al enseñar matemática, abordando temas didáctico-pedagógicos y curriculares. Este programa establece un trabajo sistemático mensual, donde algunas veces se involucra a los equipos directivos, mayormente en temas administrativos y de gestión para facilitar su implementación.

Mediante el cuestionario, se recoge información acerca del director, el equipo directivo, los compañeros de trabajo, las prácticas profesionales, el establecimiento y los programas externos de intervención que se han implementados a la fecha.

\subsection{PARTICIPANTES}

La muestra estudiada corresponde a siete establecimientos educacionales de la red de colegios de la Sociedad de Instrucción Primaria (SIP) de la Región Metropolitana. Los siete colegios son particulares subvencionados y se ubican en diferentes comunas de la 
capital. Estos entregan educación gratuita en sectores vulnerables y la población a la cual atienden, en general, es bastante homogénea. Lo que permite afirmar que los sujetos que participan del estudio y los establecimientos a los cuales pertenecen no presentan diferencias significativas que puedan distorsionar o sesgar el análisis realizado y las conclusiones que resulten del mismo.

De los siete establecimientos, cuatro participan desde el año 2013 en un programa de desarrollo profesional docente de la Universidad de Chile llamado "Activando la Resolución de Problemas en el Aula”, en el área de matemática. Los otros tres establecimientos pertenecen a la misma corporación de colegios, pero no participan, ni han participado alguna vez del programa. El número total de profesores que participa del cuestionario es de 257.

La selección de esta muestra resulta del oportuno acceso que se tiene a ella y no existe una selección intencionada que responda a requisitos especiales.

\subsection{DISEÑO DE UN INSTRUMENTO}

Esta investigación considera una etapa de diseño y validación de un instrumento para recoger información acerca de las condiciones institucionales de los establecimientos educacionales al implementar programas de asesoría externa. El diseño se realiza de acuerdo con la información que proporciona la revisión bibliográfica de las condiciones internas de los establecimientos escolares en el plano del liderazgo y el clima escolar. Además, para la construcción de los ítems se incluyen recursos de investigación e instrumentos presentados por Tschannen-Moran (2018).

Tabla 1. Dimensiones del Instrumento Cuestionario del Profesor

\begin{tabular}{|l|l|l|}
\hline Dimensión & Ítems & Descripción \\
\hline $\begin{array}{l}\text { Orientación del } \\
\text { director }\end{array}$ & $1,2,3,4,5,6,7$ & $\begin{array}{l}\text { Corresponde al estilo de liderazgo del director } \\
\text { determinado por sus prácticas, según la percepción de } \\
\text { los docentes. }\end{array}$ \\
\hline Confianza & $8,9,10,11$ & $\begin{array}{l}\text { Evalúa a la confianza que los profesores sienten hacia } \\
\text { su director, en términos de integridad, respeto, } \\
\text { competencia y estima personal por los demás. }\end{array}$ \\
\hline PEI & 12 & $\begin{array}{l}\text { Permite conocer el compromiso del docente con el } \\
\text { Proyecto Educativo Institucional de su establecimiento. }\end{array}$ \\
\hline $\begin{array}{l}\text { Satisfacción y } \\
\text { motivación }\end{array}$ & $14,15,16$ & $\begin{array}{l}\text { Permite conocer el grado de satisfacción y motivación } \\
\text { delosdocentes respectoa su trabajoen el establecimiento } \\
\text { y a su remuneración. }\end{array}$ \\
\hline $\begin{array}{l}\text { Acerca de la } \\
\text { asesoría externa }\end{array}$ & $17,18,19,20,21,22$ & $\begin{array}{l}\text { Entrega información relativa a la asesoría externa, como } \\
\text { si cumplía algún objetivo declarado en el PME o si } \\
\text { consideraba la realidad del establecimiento, por } \\
\text { ejemplo. }\end{array}$ \\
\hline
\end{tabular}

Nota: Los ítems 13 y 23 no se encuentran en una escala de tipo Likert, por lo que no son considerados en el análisis. 


\subsection{ANÁLISIS DESCRIPTIVO}

La media de las respuestas a los ítems varió entre $1.64(\mathrm{DS}=0.7)$ y 3.58 (DS=0.58). La simetría de los ítems varió entre -0.28 y 1.1 , con media $-0,94$ ( $\mathrm{DS}=0.76$ ) lo que muestra una asimetría hacia los niveles altos de la escala Likert. Por otra parte, la curtosis de los ítems varió entre -0.26 y 5.68, con media $1.26(\mathrm{DS}=2.14$ ) lo que evidencia que las respuestas a los ítems se concentran en sus valores medios, lo que sumado a la asimetría presentada demuestra la alta tasa de acuerdo que tienen los profesores con los primeros 14 ítems, que pertenecen a las primeras dimensiones del instrumento.

El análisis descriptivo mostró que los participantes tienen una percepción similar sobre el liderazgo y esta a su vez, es independiente del establecimiento en el cual trabajan. En particular, estos establecimientos son administrados por diferentes equipos directivos, en distintos puntos de la Región Metropolitana. Sin embargo, los siete establecimientos dependen de la misma corporación educacional y muchas de las decisiones tomadas al interior de ellos no dependen directamente de sus directores, sino de autoridades en cargos más altos en la corporación.

Tabla 2. Estadísticos descriptivos para los ítems de percepción de liderazgo

\begin{tabular}{|c|c|c|c|c|c|}
\hline Ítem & $\mathbf{n}$ & Media & DS & Simetría & Curtosis \\
\hline 1 & 257 & 3.36 & 0.77 & -1.37 & 2.55 \\
\hline 2 & 257 & 3.49 & 0.76 & -1.63 & 3.02 \\
\hline 3 & 257 & 3.42 & 0.88 & -2.05 & 4.86 \\
\hline 4 & 257 & 3.47 & 0.73 & -1.45 & 2.41 \\
\hline 5 & 257 & 3.22 & 0.75 & -0.61 & -0.26 \\
\hline 6 & 257 & 3.06 & 0.76 & -0.63 & 0.54 \\
\hline 7 & 257 & 3.13 & 0.77 & -0.78 & 0.76 \\
\hline 8 & 257 & 3.56 & 0.69 & -2.03 & 5.68 \\
\hline 9 & 257 & 3.49 & 0.72 & -1.61 & 3.05 \\
\hline 10 & 257 & 3.45 & 0.71 & -1.35 & 2.36 \\
\hline 11 & 257 & 1.74 & 0.93 & 1.10 & 0.47 \\
\hline 12 & 257 & 2.81 & 1.27 & -1.36 & 0.73 \\
\hline 14 & 257 & 3.58 & 0.58 & -1.64 & 5.33 \\
\hline 15 & 257 & 2.40 & 0.80 & -0.28 & -0.43 \\
\hline 16 & 257 & 1.64 & 0.70 & 0.48 & -0.30 \\
\hline
\end{tabular}




\subsection{VALIDEZ DE CONSTRUCTO: ANÁLISIS FACTORIAL EXPLORATORIO Y CONFIRMATORIO}

La muestra y sus características permiten realizar un análisis factorial exploratorio y confirmatorio ya que el valor de Kaiser-Meyer-Olkin (KMO) de adecuación muestral fue de .91, y los valores del test Bartlett's de esfericidad significativos al 99\%, con $\chi^{2}(105, n=257)=1555.05, p<.001$.

Para realizar los análisis, se dividió la muestra original $(n=257)$ en dos submuestras extraídas de forma aleatoria $\left(n_{1}=128\right.$ y $\left.n_{2}=129\right)$. La primera de ellas se utilizó para realizar el análisis exploratorio y la segunda se utilizó como muestra de validación en el análisis confirmatorio. Para los análisis basados en la Teoría de Respuesta a los Ítems, se usó toda la muestra en cada una de las sub-dimensiones resultantes.

Para realizar el AFE, se consideran los 21 ítems con la forma de extracción "ML (Máxima Probabilidad)" y rotación "VARIMAX" y luego con la forma de extracción "PA (Ejes principales)" y rotación "Promax", con el objeto de determinar posibles relaciones más débiles. Dado que las variables son ordinales se usó la matriz de correlación policórica. Para todas estas extracciones de factores se usó la función "fa" del paquete "psych" de R.

El número de factores a retener fue determinado considerando: la regla de KaiserGutman, el scree-test de Cattell y el análisis paralelo. La regla de Kaiser-Guttman (valores propios superiores a 1.00) sugirió la retención de 4 factores, y la misma sugerencia se deriva del examen del scree-test. Sin embargo, el último factor solo explicó un $2 \%$ de la varianza y su valor propio fue mayor a 1 por solo 0,06 unidades, por lo que se descartó (Timmerman y Lorenzo- Seva, 2011). Por lo anterior, se llevó a cabo un análisis paralelo optimizado (Timmerman y Lorenzo- Seva, 2011), el cual recomendó la retención de 3 factores. Si bien el modelo de 3 factores presentó buenos índices de ajustes, tanto absolutos $\left(\chi^{2}(63, n=128)=70.09, p<.25\right)$ , como relativos (TLI $=.96$; RMSEA $=.05, B I C=-225.92, R M S R=.05)$, varios ítems presentaron cargas inferiores a .4 y de forma cruzada, por lo que se procedió a eliminarlos de la escala, quedando 16 ítems. Con estos ítems se realizó nuevamente un análisis paralelo optimizado, reteniendo 3 factores los cuales presentan índices de ajustes aceptables tanto absolutos $\left(\chi^{2}(18, \mathrm{n}=128)=22.37, p<.95\right)$, como relativos $(T L I=.98 ; R M S E A=.04, B I C=-64.97, R M S R=.03)$.

Tabla 3. Índices de ajuste y varianza explicada acumulada para los métodos de extracción

\begin{tabular}{|c|c|c|c|c|c|}
\hline $\begin{array}{c}\mathrm{N}^{\mathrm{o}} \text { de Factores } \\
\text { (Extracción/Rotación) }\end{array}$ & $\chi^{2}(D F)$ & RMSEA & TLI & RMSR & $\begin{array}{c}\text { Varianza Explicada } \\
\text { Acumulada }\end{array}$ \\
\hline $\begin{array}{c}3 \\
(\mathrm{ML} / \text { VARIMAX) }\end{array}$ & $22.37(18)$ & .04 & 0.98 & .03 & .63 \\
\hline $\begin{array}{c}3 \\
(\mathrm{PA} / \mathrm{PROMAX})\end{array}$ & $23.75(18)$ & .05 & 0.97 & .03 & .63 \\
\hline
\end{tabular}

Las cargas factoriales exploratorias se muestran en la Tabla 5. 
ESTILO DE LIDERAZGO DEL DIRECTOR Y CLIMA ESCOLAR EN UN ESTABLECIMIENTO EDUCACIONAL AL IMPLEMENTAR UN PROGRAMA EXTERNO DE INTERVENCIÓN: VALIDACIÓN DE INSTRUMENTO

Tabla 4. Ajustes absolutos y relativos para ambos modelos de extracción y rotación

\begin{tabular}{|c|c|c|c|c|c|}
\hline $\begin{array}{c}\text { Modelo } \\
\text { (Función/Extracción) }\end{array}$ & $\begin{array}{c}\chi^{2} \\
(D F)(p-\text { value })\end{array}$ & CFI & TLI & RMSEA & SRMR \\
\hline Logit_ML & $28.00(24)(.26)$ & .99 & .99 & .04 & .03 \\
\hline Logit_WLS & $31.66(24)(.14)$ & .92 & .89 & .05 & .13 \\
\hline Logit_DWLS & $3.40(24)(1)$ & 1 & 1 & 0 & .03 \\
\hline Probit_ML & $28.00(24)(.26)$ & .99 & .99 & .03 & .03 \\
\hline Probit_WLS & $31.66(24)(.14)$ & .93 & .89 & .05 & .13 \\
\hline Probit_DWLS & $3.40(24)(.99)$ & 1 & 1 & 0 & .03 \\
\hline
\end{tabular}

Nota: CFI= Comparative Fit Index; TLI=Tucker-Lewis Index; RMSEA=Root Mean Square Error of Approximation; SRMSR=Standardized Root Mean Square Residual.

Con la separación de las dimensiones en los 3 factores, se procedió a realizar el Análisis Confirmatorio con la segunda muestra $(\mathrm{N}=129)$ extraída de manera aleatoria. Para esto se utilizaron tres modelos de extracción: Maximum Likelihood (ML); Weighted Least Squares (WLS); Diagonally Weighted Least Squares (DWLS), usando las funciones de enlace "probit" y "Logit", dado que las variables son de carácter ordinal. Al observar los índices de ajuste en la Tabla 3 se tiene que el modelo presenta buenos ajustes absolutos como relativos en todas las formas de determinación. La Tabla 5 muestra las cargas del análisis confirmatorio.

Tabla 5. Cargas factoriales para Análisis Factorial Exploratorio con rotación Varimax y modelo de estimación Máxima Verosimilitud, más cargas factoriales de Análisis Factorial Confirmatorio con función de enlace "probit" y modelo estimación de Máxima Verosimilitud

\begin{tabular}{|c|c|c|c|c|c|}
\hline Dimensión & Ítem & F1 & F2 & F3 & $\lambda$ \\
\hline \multirow{2}{*}{ 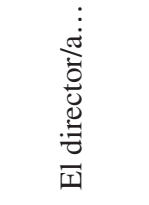 } & $\begin{array}{l}\text { 1. promueve una buena convivencia y clima en el esta- } \\
\text { blecimiento. }\end{array}$ & .87 & & & $.95 * * *$ \\
\hline & 2. nos trata con cordialidad y respeto. & .58 & & & $.91 * * *$ \\
\hline \multirow{4}{*}{ 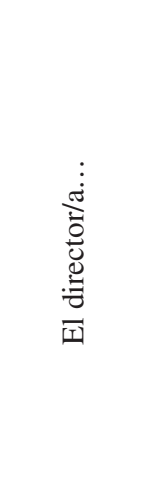 } & $\begin{array}{l}\text { 3. promueve el perfeccionamiento de los profesores } \\
\text { (becas, tiempo para capacitaciones, flexibilidad ho- } \\
\text { raria para formación). }\end{array}$ & & .50 & & $.73 * * *$ \\
\hline & $\begin{array}{l}\text { 4. genera instancias de toma de decisiones donde se in- } \\
\text { volucra la opinión de los profesores. }\end{array}$ & & .62 & & $.86^{* * * *}$ \\
\hline & $\begin{array}{l}\text { 5. procura que los profesores no se distraigan de su la- } \\
\text { bor principal (enseñanza) a través de la reducción de } \\
\text { presiones externas o administrativas. }\end{array}$ & & .55 & & $.74 * * *$ \\
\hline & $\begin{array}{l}\text { 6. promueve instancias para que los profesores } \\
\text { discutamos sobre asuntos y estrategias pedagógicas. }\end{array}$ & & .79 & & $.72 * * *$ \\
\hline
\end{tabular}


Estudios Pedagógicos XLVI Nº 2: 25-38, 2020

ESTILO DE LIDERAZGO DEL DIRECTOR Y CLIMA ESCOLAR EN UN ESTABLECIMIENTO EDUCACIONAL AL IMPLEMENTAR UN PROGRAMA EXTERNO DE INTERVENCIÓN: VALIDACIÓN DE INSTRUMENTO

\begin{tabular}{|c|c|c|c|}
\hline \multirow{3}{*}{ 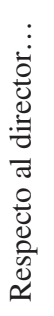 } & 8. confío en su integridad. & .55 & $.71 * * *$ \\
\hline & 9. puedo confiar en él, incluso en momentos difíciles. & .64 & $.84 * * *$ \\
\hline & 10. siento que valora mi experiencia profesional. & .77 & $.78 * * *$ \\
\hline
\end{tabular}

$* \mathrm{p}<.05 * * \mathrm{p}<.01 * * * \mathrm{p}<.001$

Nota: F1, F2, F3 = Cargas factoriales determinados con Análisis Factorial Exploratorio; $\lambda=$ Cargas factoriales determinadas con Análisis Factorial Confirmatorio.

\subsection{FIABILIDAD Y CONSISTENCIA INTERNA}

Considerando los supuestos de simetría, tau-equivalencia (Novick \& Lewis, 1967), y la falta de relación con la estructura interna del test (Sijtsma, 2009) que ha presentado el coeficiente alpha de Cronbach para medir la consistencia interna de escalas de tipo ordinal, se decidió usar el coeficiente alfa ordinal, considerando las cargas factoriales de los ítems (Zumbo, Gadermann, y Zeisser, 2007), el coeficiente theta ordinal, dado los mayores autovalores de la matriz de correlaciones policóricas y el coeficiente Omega (McDonald, 1999). La Tabla 6 muestra que los valores de los coeficientes son todos superiores .78, lo que confirma la fiabilidad tanto para cada uno de los factores como para la escala en general.

Tabla 6. Coeficientes de fiabilidad alpha ordinal, theta ordinal y omega para cada factor y para la escala completa

\begin{tabular}{|c|r|r|c|}
\hline Factor & $\alpha_{O R D}$ & $\theta_{O R D}$ & $\omega$ \\
\hline 1 & .90 & .99 & .91 \\
\hline 2 & .87 & .76 & .88 \\
\hline 3 & .88 & .67 & .92 \\
\hline$\alpha_{O R D}(E C)$ & \multicolumn{3}{|c|}{.93} \\
\hline$\theta_{O R D}(E C)$ & \multicolumn{3}{|c}{.95} \\
\hline$\omega(E C)$ & \multicolumn{3}{|c}{} \\
\hline
\end{tabular}

Nota: $\alpha_{O R D}=$ Coeficiente Alpha ordinal; $\theta_{\text {ORD }}=$ Coeficiente Theta ordinal; $\omega=$ Coeficiente Omega; $\alpha_{O R D}(E C)=$ Coeficiente Alpha ordinal Escala Completa; $\theta_{\mathrm{ORD}}(\mathrm{EC})=$ Coeficiente Theta ordinal Escala Completa; $\omega$ (EC) =Coeficiente Omega Escala Completa. 


\subsection{CALIBRACIÓN}

Habiendo comprobado la unidimensionalidad e independencia local de cada uno de los factores que componen la escala sobre percepción de liderazgo se llevó a cabo un análisis de calibración de ítems para cada uno de los factores, usando el modelo Respuesta Gradual de Samejima (1969) bajo la Teoría de Respuesta al Ítem (TRI). Este modelo se caracteriza por determinar la probabilidad de que un sujeto, marque una categoría $\beta_{i}$ o una superior versus que marque una categoría inferior $\beta_{i-1}$. Las estimaciones se hicieron usando la función "grm" del paquete "ltm" de R (Rizopoulos, 2006).

Tabla 7. Parámetros del modelo GRM

\begin{tabular}{|c|c|c|c|c|c|c|c|}
\hline Factor & Ítem & $\beta_{1}$ & $\beta_{2}$ & $\beta_{3}$ & $\alpha$ & INF \\
\hline \multirow{3}{*}{1} & 1 & -11.40 & -6.89 & 0.03 & 4.67 & 13.6 \\
\cline { 2 - 7 } & 2 & -9.48 & -5.48 & -1.26 & 3.66 & 10.22 \\
\hline \multirow{3}{*}{2} & 3 & -6.64 & -4.06 & -0.66 & 2.52 & 6.4 \\
\cline { 2 - 7 } & 4 & -10.28 & -4.71 & 0.98 & 4.53 & 13.3 \\
\cline { 2 - 7 } & 5 & -6.28 & -2.81 & 1.68 & 2.67 & 7.36 \\
\cline { 2 - 7 } & 6 & -5.77 & -2.92 & 1.20 & 2.52 & 6.68 \\
\hline \multirow{3}{*}{3} & 7 & -10.79 & -7.37 & -1.43 & 3.98 & 11.17 \\
\cline { 2 - 8 } & 8 & -11.22 & -7.38 & -1.15 & 4.82 & 13.76 \\
\cline { 2 - 8 } & 9 & -8.33 & -4.87 & -0.41 & 2.95 & 8.13 \\
\hline
\end{tabular}

Nota: $\beta_{1}=$ Umbral de la habilidad latente entre la categoría respuesta nunca y rara vez; $\beta_{2}=$ Umbral de la habilidad latente entre la categoría respuesta rara vez y casi siempre; $\beta_{3}=$ Umbral de la habilidad latente entre la categoría respuesta casi siempre y siempre; $\alpha=$ discriminación del ítem; INF=Valor de la función de información.

Los parámetros “ $\alpha$ ” están entre 2.52 y 4.82, con media $\mathrm{M}=3.59$ ( $\mathrm{SD}=0.95)$, por lo que los ítems de percepción de liderazgo discriminan bien, según los parámetros de Baker (2001). Los umbrales $\beta_{3}$ van desde -1.23 hasta 1.68, con media $\mathrm{M}=-0,11$ ( $\left.\mathrm{SD}=1.15\right)$. Los umbrales $\beta_{2}$ van desde -7.38 hasta -2.81 , con media $\mathrm{M}=-5.17$ ( $\left.\mathrm{SD}=1.77\right)$. Los umbrales $\beta_{1}$ van desde -11.40 hasta -5.77 , con media $\mathrm{M}=-8.91$ ( $\mathrm{SD}=2.22$ ). De lo anterior, todos los umbrales respetan el supuesto de orden, y muestran que las respuestas de los profesores están cargadas a los niveles Likert más altos en cada uno de los ítems. Por otra parte, la información de los ítems estuvo entre 6.40 y 13.76, con media 10.07 ( $\mathrm{SD}=3.04$ ), teniendo una información total de 90.62. En cada uno de los factores, ninguno de los residuos de las combinaciones posibles entre los ítems excedió los umbrales. Por otra parte, al comparar los modelos ajustados con modelos con el índice de discriminación contraído en cada uno de los factores usando el Test de Razón de Verosimilitud, se tiene que éste último no ajustó mejor que el primero en ninguno de los factores de la escala $\left(F 1: \chi^{2}(1)=8.4, p<.02 ; F 2: \chi^{2}(3)=8.15, p<.04 ; F 3: \chi^{2}(2)=3.82, p<.01\right)$. 
Estudios Pedagógicos XLVI N 2: 25-38, 2020

ESTILO DE LIDERAZGO DEL DIRECTOR Y CLIMA ESCOLAR EN UN ESTABLECIMIENTO EDUCACIONAL AL IMPLEMENTAR UN PROGRAMA EXTERNO DE INTERVENCIÓN: VALIDACIÓN DE INSTRUMENTO

\section{INTERPRETACIONES, DISCUSIÓN Y CONCLUSIONES}

A través del instrumento se buscaba recoger información sobre las condiciones institucionales de los establecimientos educacionales al implementar programas de asesoría externa. Para esto, las dimensiones analizadas fueron el grado de confianza entre los integrantes de la comunidad escolar, el estilo de liderazgo del director, la satisfacción y motivación docente y las características de la estructura de la organización. Luego de la aplicación en una muestra de siete colegios, se analizó la capacidad del instrumento para levantar información considerando diferentes aspectos de este, los cuales se detallan a continuación.

Dados los índices de fiabilidad, confiabilidad y calibración que ha mostrado el instrumento en una muestra de 257 profesores de siete colegios de la Región Metropolitana, se puede afirmar que el instrumento puede ser usado con total confianza en el estudio de la percepción de liderazgo por los profesores en establecimientos educacionales, más aun considerando que los análisis llevados a cabo para su validez tienen mejores aproximaciones a escalas de tipo ordinal, que los llevados a cabo en otras investigaciones, donde se ha omitido el hecho que las escalas usadas sean de tipo politómica.

En particular, la primera dimensión, Orientación del director, se dividió en dos factores, el primero, compuesto por 2 indicadores que explican el $23 \%$ de la varianza, se relaciona tanto con el clima que genera el director en el establecimiento como con el trato que tiene hacia los docentes en la relación de persona a persona. El factor 2, compuesto por 4 indicadores que explican el $21 \%$ de la varianza, representa las prácticas del director que se relacionan con el ejercicio docente. Incluyendo el grado de consideración que puede tener por los docentes en la toma de decisiones o en las acciones con las que busca reducir presiones externas. La segunda dimensión, Confianza, no se dividió, resultando significativos los 4 indicadores que la componen, los cuales explican un 19\% de la varianza total. Estos tienen relación con la confianza que los profesores tienen en el director, en base a su competencia, estima personal por los otros, integridad y respeto (Razeto, 2016; Tshcannen-Moran, 2009, 2013). De esta forma, el análisis factorial mostró que el instrumento es capaz de describir tres condiciones internas en un establecimiento educacional: el clima generado por el director, el estilo de las prácticas del director en relación con sus profesores y la confianza de los profesores en el director.

La estructura de la escuela, de acuerdo con la literatura, es una consecuencia de las prácticas que lleva a cabo el director, junto con el grado en confianza de las relaciones interpersonales de la comunidad escolar (Hoy y Sweetland, 2000, 2001; Ríos \& Villalobos, 2016; López et al., 2012; Tschannen-Moran, 2004, 2009, 2013). Por lo que este instrumento también podría indicar con bastante precisión la estructura adoptada por la organización escolar, al entregar información sobre las prácticas del líder y la confianza.

Entre las debilidades de la validez, se pueden mencionar los siguientes puntos: a) dada las características de los datos, no fue posible aplicar análisis estadísticos de temporalidad, b) no fue posible profundizar la validez externa, consecuencial y nomológica, c) y tampoco fue posible aplicar análisis de varianza tanto en los grupos como en el tiempo. Claro está que estas debilidades, también se vuelven oportunidades para realizar futuras investigaciones que permitan profundizar en el análisis de las condiciones internas de los establecimientos educacionales al implementar programas externos de intervención.

Pese a que la literatura (López et al., 2012; Tschannen-Moran, 2009, 2013) indica que la satisfacción y motivación de los docentes es un elemento clave que resulta del clima organizacional que genera el director y del grado de confianza que los profesores tienen 
en él, ninguno de los ítems de la dimensión Satisfacción y Motivación resultó significativo para explicar alguna condición interna de la organización.

Lo anterior puede ser una consecuencia de la distribución jerárquica y estructura de administración de las escuelas estudiadas, ya que el director de la escuela no es quien concentra el poder de decisión, sino los agentes externos, que son elegidos por las autoridades de la corporación educacional.

En este sentido es importante señalar que este modelo administrativo está presente en la mayoría de los establecimientos educacionales del país, donde son los sostenedores, en el caso de las escuelas municipales, fundaciones y corporaciones; y los asesores pedagógicos, en el caso de las escuelas particulares, los que finalmente deciden sobre muchos de los aspectos de las escuelas, por sobre los equipos directivos de las mismas. Esto significa que, aunque los directores promueven un clima que favorezca el aprendizaje y el desarrollo del ejercicio docente en sus escuelas, la mayoría de sus profesores no se sienten satisfechos y motivados por el trabajo que realizan, porque las condiciones finales que afectan su trabajo no dependen directamente del director de su escuela.

Dado lo anterior, se propone como proyección de este trabajo analizar la información que recoge el instrumento diseñado en otro tipo de establecimientos, como escuelas rurales, por ejemplo, u otras organizaciones educativas diferentes donde los líderes que toman las decisiones sean cercanos al grupo de profesores e integrantes diligentes de la comunidad escolar.

\section{REFERENCIAS BIBLIOGRÁFICAS}

Bellei, C., Osses, A. \& Valenzuela, J. (2010). Asistencia técnica educativa: De la intuición a la evidencia. Santiago: Editorial Ocholibros.

Espíndola, V. \& Silva, M. (2009). Competencias del sostenedor para una efectiva gestión del mejoramiento educativo en el nivel local: una propuesta. Foco Educación, CPCE / Expansiva UDP, 2-31. Recuperado desde http://www.expansiva.cl/media/en_foco_edu/ documentos/11122009123954.pdf

Hernández-Castilla, R., Murillo, F. J. \& Martínez-Garrido, C. (2013). Factores de ineficacia escolar School ineffectiveness factors. REICE. Revista Iberoamericana Sobre Calidad.

Hoy, W. K. \& Sweetland, S. R. (2000). Bureaucracies that work: Enabling not coercive. Journal of School Leadership, 10(6), 525-541.

(2001). Designing better schools: The meaning and nature of enabling school structure. Educational Administration Quarterly, 37(3), 296-321.

López, V., Ahumada, L., Olivares, R. \& González, Á. (2012). Escala de medición del aprendizaje organizacional en centros escolares. Psicothema, 24(2), 323-329.

López Yáñez, J. (2010). Sostenibilidad de la innovación en los centros escolares: sus bases institucionales. Profesorado: Revista de Curriculum y Formación Del Profesorado, 14(1), 9-28.

Marchant, J. P. \& Salin, A. (2009). Experiencias de intervención institucional en escuelas vulnerables en latinoamérica: una revisión desde la mejora de la calidad. Revista Iberoamericana Sobre Calidad, Eficacia y Cambio En Educación.

Razeto Pavez, A. (2016). Confianza interpersonal entre los miembros de una escuela: Valor básico y olvidado por las reformas educativas. Revista Electrónica "Actualidades Investigativas en Educación”, 16(1), 1-22.

Ríos, D. \& Villalobos, P. (2016). Mejora educativa a partir de asesoría externa: el complejo camino hacia la sostenibilidad. Estudios Pedagógicos (Valdivia). 42(2), 315-330. https://doi.org/10.4067/ S0718-07052016000200018 
Estudios Pedagógicos XLVI Nº 2: 25-38, 2020

ESTILO DE LIDERAZGO DEL DIRECTOR Y CLIMA ESCOLAR EN UN ESTABLECIMIENTO EDUCACIONAL AL

IMPLEMENTAR UN PROGRAMA EXTERNO DE INTERVENCIÓN: VALIDACIÓN DE INSTRUMENTO

Sotomayor, C. (2006). Programas públicos de mejoramiento de la calidad de escuelas básicas en contextos urbanos vulnerables: evolución y aprendizajes de sus estrategias de intervención (1990 -2005). Pensamiento Educativo.

Tschannen-Moran, M. (2004). Trust matters: Leadership for successful schools. San Francisco: Jossey-Bass.

. (2009). Fostering Teacher Professionalism in Schools: The Role of Leadership Orientation and Trust. Educational Administration Quarterly, 45(2), 217-247.

. (2013). Trust matters: leadership for successful schools. Journal of Educational Administration.

. (2018). Survey Instruments to Help You in Your Investigations of Schools. New York, EU.: Megan Tschannen-Moran's Web Site. Recuperado de: http://wmpeople.wm.edu/site/page/ $\mathrm{mxtsch} /$ researchtools

Valenzuela, J. \& Allende, C. (2014). Trayectorias de mejoramiento en el sistema educacional chileno. Apuntes sobre mejoramiento escolar. UNICEF-CIAE, 1, 1-15.

Timmerman, M. E. y Lorenzo-Seva, U. (2011). Dimensionality assess-ment of ordered polytomous items with parallel analysis. Psychological Methods, 16, 209-220. doi: 10.1037/ a0023353

Novick, M. R. \& Lewis, C. (1967). Coefficient alpha and the reliability of composite measurements. Psychometrika, 32, 1-13.

Samejima, F. (1969). Estimation of latent trait ability using a response pattern of graded scores. Psychometrika Monograph, (1), 17. doi: 10.1002/j.2333-8504.1968.tb00153.x

Zumbo, B. D., Gadermann, A. M. y Zeisser, C. (2007). Ordinal versions of coefficients alpha and theta for Likert rating scales. Journal of modern applied statistical methods, 6(1), 21-29. DOI: $10.22237 / \mathrm{jmasm} / 1177992180$.

McDonald, R. P. (1999). Test theory: A unified treatment. Mahwah, NJ: Lawrence Erlbaum Associates.

Rizopoulos, D. (2006). ltm: An R package for latent variable modelling and item response theory analyses. Journal of Statistical Software, 17(5), 1-25. Obtenido de https://core.ac.uk/download/ pdf/6305163.pdf. 